\title{
Active Oxygen Target for Studies in Nuclear Astrophysics with Laser Compton Backscattered $\gamma$-ray Beams
}

\author{
Robert Ajvazyan ${ }^{1}$, John R. M. Annand ${ }^{2}$, Dimiter L. Balabanski ${ }^{3}$, Nersik Grigoryan ${ }^{1}$, \\ Vanik Kakoyan ${ }^{1}$, Patrik Khachatryan ${ }^{1}$, Vachik Khachatryan ${ }^{1}$, Kenneth Livingston ${ }^{2}$, \\ Rachel Montgomery ${ }^{2}$, Henrik Vardanyan ${ }^{1}$, Branislav Vlahovic ${ }^{4}$, Simon Zhamkochyan ${ }^{1}$ and \\ Amur Margaryan ${ }^{1, *}$ \\ 1 A.I. Alikhanyan National Science Laboratory, 2 Alikhanyan Bros. Str., Yerevan 0036, Armenia; \\ ayvazyan@mail.yerphi.am (R.A.); nerses@mail.yerphi.am (N.G.); kakoyan@mail.yerphi.am (V.Ka.); \\ patrick@mail.yerphi.am (P.K.); vachik@mail.yerphi.am (V.Kh.); henrik@mail.yerphi.am (H.V.); \\ szh@mail.yerphi.am (S.Z.) \\ 2 School of Physics \& Astronomy, University of Glasgow, Scotland G128QQ, UK; \\ john.annand@glasgow.ac.uk (J.R.M.A.); Kenneth.Livingston@glasgow.ac.uk (K.L.); \\ Rachel.Montgomery@glasgow.ac.uk (R.M.) \\ 3 ELI-NP, Horia-Hulubay National Institute of Physics and Nuclear Engineering, Bucharest 077125, Magurele, \\ Romania; dimiter.balabanski@eli-np.ro \\ 4 Department Science and Technology, North Carolina Central University, Durham 27707, NC, USA; \\ vlahovic@nccu.edu \\ * Correspondence: mat@mail.yerphi.am; Tel.: +374-1034-1500
}

Received: 30 January 2018; Accepted: 16 May 2018; Published: 20 May 2018

Abstract: An active target is being developed to be used in low-energy nuclear astrophysics experiments. It is a position- and time-sensitive detector system based on the low-pressure Multi Wire Proportional Chamber (MWPC) technique. Methylal $\left(\left(\mathrm{OCH}_{3}\right)_{2} \mathrm{CH}_{2}\right)$, at a pressure of a few Torr, serves as the working gas for MWPC operation, and in addition, the oxygen atoms of the methylal molecules serve as an experimental target. The main advantage of this new target detector system is that it has high sensitivity to the low-energy, highly-ionizing particles produced after photodisintegration of ${ }^{16} \mathrm{O}$ and insensitivity to $\gamma$-rays and minimum ionizing particles. This allows users to detect only the products of the nuclear reaction of interest. The threshold energies for detection of $\alpha$ particles and ${ }^{12} \mathrm{C}$ nuclei are about $50 \mathrm{keV}$ and $100 \mathrm{keV}$, respectively. The main disadvantage of this detector is the small target thickness, which is around a few tens of $\mu \mathrm{g} / \mathrm{cm}^{2}$. However, reasonable luminosity can be achieved by using a multimodule detector system and an intense, Laser Compton Backscattered (LCB) $\gamma$-ray beam. This paper summarizes the architecture of the active target and reports test results of the prototype detector. The tests investigated the timing and position resolutions of $30 \times 30 \mathrm{~mm}^{2}$ low-pressure MWPC units using an $\alpha$-particle source. The possibility of measuring the ${ }^{16} \mathrm{O}(\gamma$, $\alpha)^{12} \mathrm{C}$ cross-section in the $8-10 \mathrm{MeV}$ energy region by using a LCB $\gamma$-ray beam is also discussed. A measurement of the ${ }^{16} \mathrm{O}(\gamma, \alpha){ }^{12} \mathrm{C}$ cross-section will enable the reaction rate of ${ }^{12} \mathrm{C}(\alpha, \gamma){ }^{16} \mathrm{O}$ to be determined with significantly improved precision compared to previous experiments.

Keywords: nuclear astrophysics; active target; gaseous detectors

\section{Introduction}

Thermonuclear burning in stars is responsible for the synthesis of most chemical elements heavier than helium in the universe. The ${ }^{4} \mathrm{He}(\alpha \alpha, \gamma){ }^{12} \mathrm{C}$ and ${ }^{12} \mathrm{C}(\alpha, \gamma){ }^{16} \mathrm{O}$ reactions are two of the most 
important fusion processes in astrophysics [1]. These reactions essentially govern the helium burning phase, and their rates determine the ratio of ${ }^{12} \mathrm{C}:{ }^{16} \mathrm{O}(\mathrm{C} / \mathrm{O})$ in the "ashes" of the helium burning process. Consequently, these reactions strongly influence the subsequent nucleosynthesis processes for massive stars and their final nucleosynthesis yields [2]. Stellar evolution models in which nuclear reaction networks are implemented in detail do not yield consistent results yet, and the ${ }^{12} \mathrm{C}(\alpha, \gamma){ }^{16} \mathrm{O}$ cross-section is one of the most important nuclear physics uncertainties still to be resolved.

There are currently two experimental approaches under consideration for measurement of the inverse ${ }^{16} \mathrm{O}(\gamma, \alpha){ }^{12} \mathrm{C}$ reaction, which is more accessible than the direct ${ }^{12} \mathrm{C}(\alpha, \gamma){ }^{16} \mathrm{O}$ reaction. One is based on the bubble chamber technique and a bremsstrahlung photon beam [3]; the other employs an Optical Time Projection Chamber (O-TPC) [4] and a Laser Compton Backscattered (LCB) $\gamma$-ray beam [5-7].

The reciprocity theorem of nuclear reactions relates the cross-sections of forward- and time-reversed nuclear processes. It relates the cross-section of an $(\alpha, \gamma)$ process to that of the time-reversed $(\gamma, \alpha)$ reaction:

$$
\omega_{A} \frac{\sigma_{A}(\alpha, \gamma)}{l_{A}}=\omega_{B} \frac{\sigma_{B}(\gamma, \alpha)}{l_{B}}
$$

where $\sigma_{A}(\alpha, \gamma), \sigma_{B}(\gamma, \alpha)$ are the cross-sections of the direct ${ }^{12} \mathrm{C}(\alpha, \gamma){ }^{16} \mathrm{O}$ and inverse ${ }^{16} \mathrm{O}(\gamma, \alpha){ }^{12} \mathrm{C}$ reactions, $l_{A}$ and $l_{B}$ are the channel wavelengths for capture and photodisintegration, and $\omega_{A}$ and $\omega_{B}$ are the respective spin factors. In the energy region discussed here, i.e., $E \gamma=8.0-8.5 \mathrm{MeV}$, the transformation factor provides a gain of $\sim 50$ in cross-section. Thus, at an equivalent center of mass energy of $\sim 0.9 \mathrm{MeV}$, the direct $(\alpha, \gamma)$ cross-section is $\sim 60 \mathrm{pb}$, while the inverse $(\gamma, \alpha)$ cross-section at $\sim 8 \mathrm{MeV}$ is $3 \mathrm{nb}$. The photodisintegration of ${ }^{16} \mathrm{O}$ at $8.0 \mathrm{MeV}$ produces ${ }^{4} \mathrm{He}$ and ${ }^{12} \mathrm{C}$ with energies 675 and $225 \mathrm{keV}$, respectively, flying back-to-back, which aids the positive identification of this channel.

We present a new active target for measuring the cross-section of the ${ }^{16} \mathrm{O}(\gamma, \alpha)^{12} \mathrm{C}$ reaction, using an LCB $\gamma$-ray beam of energy $E_{\gamma}=8-10 \mathrm{MeV}$. It is a position- and time-sensitive detector system based on low-pressure Multi Wire Proportional Chamber (MWPC) technology. This enables the trajectories and velocities of ${ }^{4} \mathrm{He}$ and ${ }^{12} \mathrm{C}$ fragments of a few hundred $\mathrm{keV}$ energy to be determined. The experimental method is described in Section 2, while Section 3 summarizes the experimental setup and the results of test measurements of timing and position resolution using $\alpha$-particles from a ${ }^{239} \mathrm{Pu}$ source.

\section{Methods}

The new method is based on two technologies: (1) an active target based on low-pressure MWPC, and (2) a gamma-ray beam from backscattering of laser photons from several hundred $\mathrm{MeV}$ electrons [3]. The latter produces an intense monochromatic gamma-ray beam with small angular divergence $\left(<2 \times 10^{-4} \mathrm{rad}\right)[4,5]$. Methylal $\left(\left(\mathrm{OCH}_{3}\right)_{2} \mathrm{CH}_{2}\right)$ and hexane $\left(\mathrm{C}_{6} \mathrm{H}_{14}\right)$, at a pressure of a few Torr, serve as the filling gases for operation of the MWPC. Operation with hexane is intended for evaluation of the background from carbon nuclei in methylal. The proposed active-target system employs a gas pressure of 3-9 Torr to detect low-energy nuclear fragments and determine their trajectories and velocities. The low pressure renders it very insensitive to minimum ionizing particles and $\gamma$-rays, but the disadvantage of this is low luminosity due to the small density of target atoms. Laser backscattering delivers a highly-directed, pencil-like photon beam so that this drawback can be mitigated by using a multimodular active-target system with sufficient total length to provide the necessary luminosity for the experiment. For example, at ELI-NP, the angular divergence of the LCB $\gamma$-ray beam is expected to be less than $0.2 \times 10^{-4} \mathrm{rad}$, which means that over a $10 \mathrm{~m}$ distance the transverse size of the beam will increase by only $2 \mathrm{~mm}$. Therefore, the active-target system could, in principle, consist of a stack of some hundreds of modules with a total length equal to $10 \mathrm{~m}$.

A schematic drawing of the prototype, active-target module is shown in Figure 1. It consists of four low-pressure chamber units (1, 2, 3 and 4), which form two symmetric arms. The MWPC units are windowless in order to present the minimum amount of material to the incident, low-energy nuclear 
fragments. The geometrical acceptance of the active target depends on the L1, L2 and L3 dimensions, where the optimal sizes will be determined in future Monte Carlo and experimental studies. In the present study, we used prototype MWPC units with an active area of $30 \times 30 \mathrm{~mm}^{2}$.

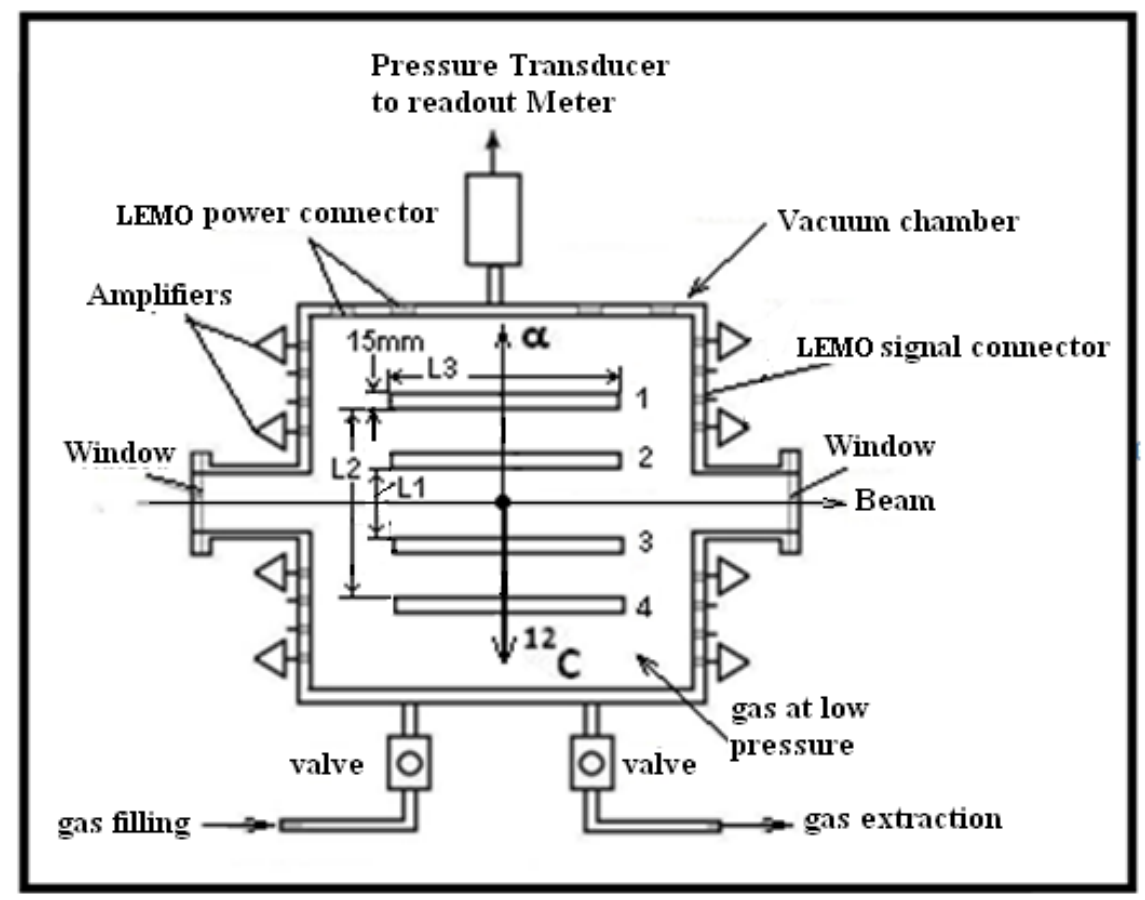

Figure 1. Schematic of the active target: Multi Wire Proportional Chamber (MWPC) units 1, 2, 3, 4.

Figure 2 shows schematically the structure of a single MWPC chamber. It consists of five wire planes supported by glass textolite (epoxy) frames with a thickness of $3 \mathrm{~mm}$, which is the plane spacing. A time signal is extracted from the central anode plane, where all anode wires are grouped together. This is placed between two cathode planes, which have their wires oriented at an angle of $90^{\circ}$ with respect to each other.



Figure 2. Schematic diagram of the single unit of MWPC.

The positive signals induced on the cathodes are used for the position $(x, y)$ readout, one coordinate from each plane. The two outer wire planes function as guards against electrons produced by ionization taking place outside of the chamber region and as additional electrodes to provide double-step amplification. All planes employ a wire spacing of $1 \mathrm{~mm}$. The anode plane is composed of $20 \mu \mathrm{m}$ diameter gold-plated tungsten wire, while the cathode and guard planes are composed of $40 \mu \mathrm{m}$ 
diameter copper-beryllium wires. The cathode wires are connected-in groups of three-to tapped delay lines that provide $2 \mathrm{~ns} / \mathrm{tap}$ and a maximum delay time of $20 \mathrm{~ns}$, equivalent to $3 \mathrm{~cm}$ distance. In our test studies, we used the time difference between the anode pulse and the induced cathode pulse after propagation to the end of a delay line. Two position-sensitive MWPC units (Figure 1), separated by $5.5 \mathrm{~cm}$, are situated on each side of the beam, but the final separation will be optimized in future detailed Monte Carlo simulations. The MWPCs provide position and time information so that trajectories and velocities of incident particles can be determined to enable separation of low-energy ${ }^{16} \mathrm{O}(\gamma, \alpha)^{12} \mathrm{C}$ photodisintegration from background reactions. The background is mainly due to production of electron-positron pairs and due to photodisintegration of ${ }^{16} \mathrm{O}$ and ${ }^{12} \mathrm{C}$ nuclei initiated by the high-energy bremsstrahlung photons of the $\gamma$-ray beam. In Figure 1, alpha particles are detected by MWPC units 1 and 2, while ${ }^{12} \mathrm{C}$ nuclei are detected by units 3 and 4 .

The MWPCs are mounted in a chamber (see Figure 1), which has windows for beam entrance and exit. Connectors attached to the walls of the vacuum chamber provide access to electronics outside the target chamber. The fast, ultralow noise amplifiers of the nanosecond signals from the anode and cathode planes were developed at the Alikhanyan National Science Laboratory [8] and are mounted outside the chamber. The chamber is equipped with valves for gas handling and two pressure gauges, and the gas inlet is connected to a reservoir of liquid methylal $\left(\left(\mathrm{OCH}_{3}\right)_{2} \mathrm{CH}_{2}\right)$ or hexane $\left(\mathrm{C}_{6} \mathrm{H}_{14}\right)$ [9-11] via a reducing valve. After evacuation to a pressure of $10^{-3}$ Torr, it was filled with a 2-9 Torr of methylal (hexane). The methylal (hexane) gas serves as an ionization medium for MWPC operation, and in addition, the oxygen atoms of the methylal molecules serve as the ${ }^{16} \mathrm{O}$ target. The number of oxygen atoms in the target is given by $N_{\mathrm{O}}=N_{\mathrm{A}} \times \rho \times p \times L \times n / A$, where $\mathrm{L}$ is the target thickness, $\mathrm{n}$ is the number of oxygen atoms per $\mathrm{C}_{3} \mathrm{O}_{2} \mathrm{H}_{8}$ molecule $(n=2), \rho$ is the density of the methylal gas at $20^{\circ} \mathrm{C}\left(\rho=0.0042 \mathrm{mg} /\right.$ Torr $\left./ \mathrm{cm}^{3}\right), \mathrm{p}$ is the pressure in Torr, $\mathrm{N}_{\mathrm{A}}$ is Avogadro's number, and $\mathrm{A}$ is the molecular weight of $\mathrm{C}_{3} \mathrm{O}_{2} \mathrm{H}_{8}$ in a.m.u. $(A=76)$. For a single module with $L=3 \mathrm{~cm}$ and $p=3$ (6) Torr, we have $0.0126(0.0252) \mathrm{mg} / \mathrm{cm}^{2}$ methylal, which results in $N_{\mathrm{O}}=6 \times 10^{17}\left(1.2 \times 10^{18}\right)$ atoms $/ \mathrm{cm}^{2}$.

Using a multimodule active-target system consisting of some hundreds of modules, the number of oxygen atoms can be increased by over two orders of magnitude. This is presented schematically in Figure 3. Assuming that the number of oxygen atoms seen by the beam is $3 \times 10^{20}$ atoms $/ \mathrm{cm}^{2}$, the detection efficiency for the ${ }^{16} \mathrm{O}(\gamma, \alpha){ }^{12} \mathrm{C}$ reaction (geometrical acceptance included) is $30 \%$, the $\gamma$-ray intensity is $5 \times 10^{9}$ photons / $\mathrm{s}$ [7], and the cross-section of the ${ }^{16} \mathrm{O}(\gamma, \alpha){ }^{12} \mathrm{C}$ reaction is $3 \mathrm{nb}$, then the ${ }^{16} \mathrm{O}(\gamma, \alpha)^{12} \mathrm{C}$ reaction rate is equal to $3 \times 10^{20} \times 5 \times 10^{9} \times 0.3 \times 3 \times 10^{-33}=0.00135 \mathrm{event} / \mathrm{s}$.

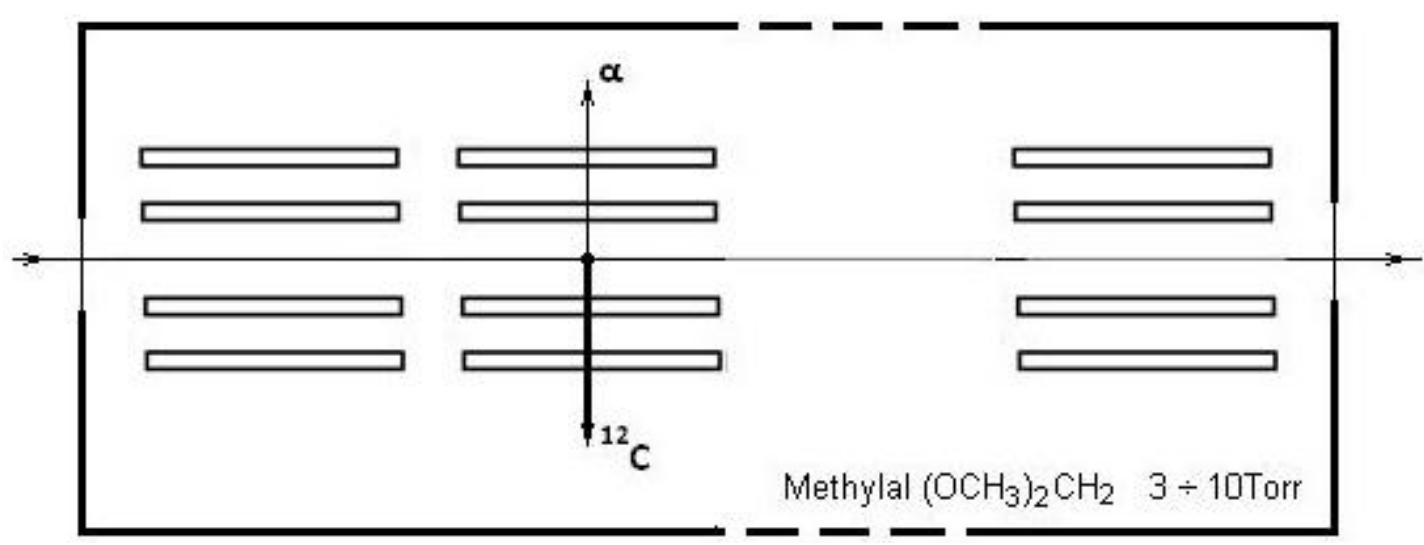

Figure 3. Schematic of the multimodule active target.

\section{Results}

Timing information is an important consideration when using the active target. Firstly, the real coincidence between modules and beam bunches must be separable event-by-event. At ELI-NP, the separation between photonbeam bunches is $16 \mathrm{~ns}$, so that a timing resolution of $\sim 1 \mathrm{~ns}$ is necessary 
for separating the events from different bunches. Secondly, the time-of-flight between the low-pressure MWPC units can be used to determine the velocities of the produced alpha particles and ${ }^{12} \mathrm{C}$ nuclei, thus providing an additional criterion to separate real ${ }^{16} \mathrm{O}(\gamma, \alpha){ }^{12} \mathrm{C}$ events from the background. Good position resolution is needed to identify back-to-back fragments and to separate these from the huge amount of electromagnetic background (the electron-positron pair production cross-section in this energy region is about $100 \mathrm{mb}$, i.e., $10^{8}$ times larger than the photodisintegration cross-section of $\left.{ }^{16} \mathrm{O}\right)$.

A test setup (Figure 4) was constructed to investigate the operational modes of the low-pressure MWPC units and to measure the timing and position-sensing characteristics. It used a $0.14 \mathrm{mg} / \mathrm{cm}^{2}$ thick, $30 \times 30 \mathrm{~mm}^{2}{ }^{239} \mathrm{Pu} \alpha$-particle source which has three lines: $11 \%-5.099 \mathrm{MeV}, 20 \%-5.137 \mathrm{MeV}$ and $69 \%-5.150 \mathrm{MeV}$. Two units, MWPC1 and MWPC2, separated by $5.5 \mathrm{~cm}$, detect the $\alpha$-particles, which are collimated by mylar absorbers with a $1 \mathrm{~mm}$ diameter hole in the center. With this setup, the time and position resolutions and $\mathrm{d} E / \mathrm{d} x$ distributions in the MWPCs were measured for incident $\alpha$-particles. Data was also obtained for reduced $\alpha$-particle energies, obtained by placing a polyethylene absorber between the Pu-239 source and the first Mylar collimator. Data acquisition was realized on a CAMAC system using Phillips 715 constant fraction timing discriminators and LeCroy 2228A time-to-digital converters that were set to a nominal resolution of $250 \mathrm{ps} / \mathrm{channel}$.

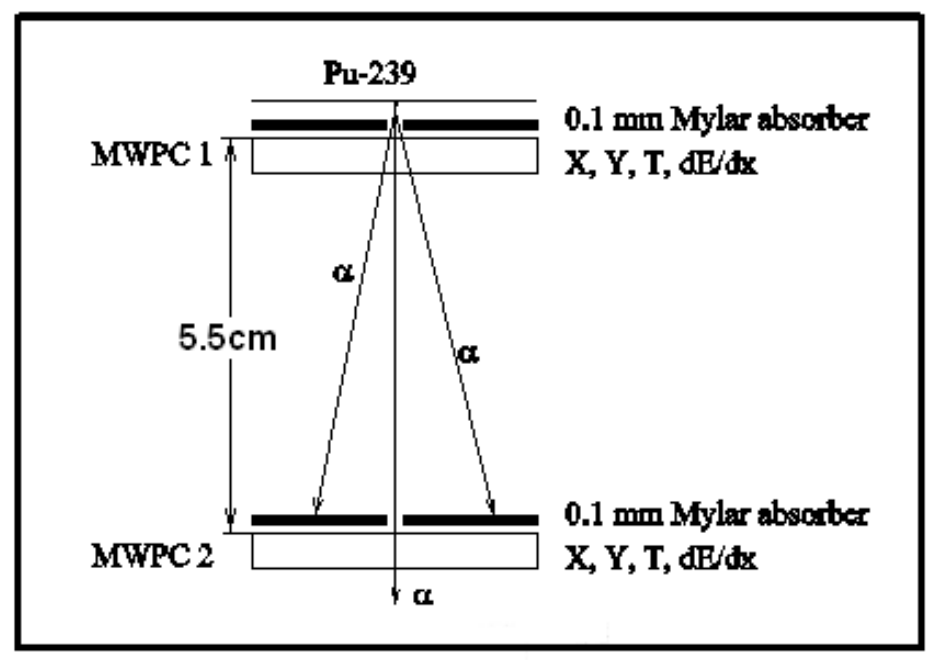

Figure 4. Schematic representation of the test setup.

The low-pressure MWPC system can be operated in the so-called single-step and double-step modes ([9-11] and references therein). In the case of single step, the typical potentials applied to the anode, cathode and guard planes are $U_{\mathrm{a}}=+400, U_{\mathrm{c}}=-100$ and $U_{\mathrm{g}}=0 \mathrm{~V}$, respectively, and the resulting signals from the $\alpha$-particles have few tens of $\mathrm{mV}$ amplitude. In the case of double-step, the typical potentials are $U_{\mathrm{a}}=+300, U_{\mathrm{c}}=0$ and $U_{\mathrm{g}}=-300 \mathrm{~V}$ and amplitudes of the resulting signals are increased by an order of magnitude, compared to the single-step case. The traces of typical $\alpha$-particles signals from the anode and cathode planes in double-step mode are displayed in Figures 5 and 6, obtained, respectively without and with a $24 \mu \mathrm{m}$ thick polyethylene absorber inserted. The time-difference distributions for signals from the anode planes of MWPC1 and MWPC2 are shown in Figure 7 (without absorber) and Figure 8 (with absorber). A Gaussian fit to the time-difference distribution of Figure 7 has a standard deviation of $638 \mathrm{ps}$. Assuming that the timing resolutions of the two contributing MWPC units are equal, the time resolutions of the individual MWPC units are equal to $638 / 2^{1 / 2} \approx 450 \mathrm{ps}$. From the distribution presented in Figure 8, one can determine the energy distribution of $\alpha$-particles after passing through the $24 \mu \mathrm{m}$ polyethylene foil. This distribution is presented in Figure 9. 


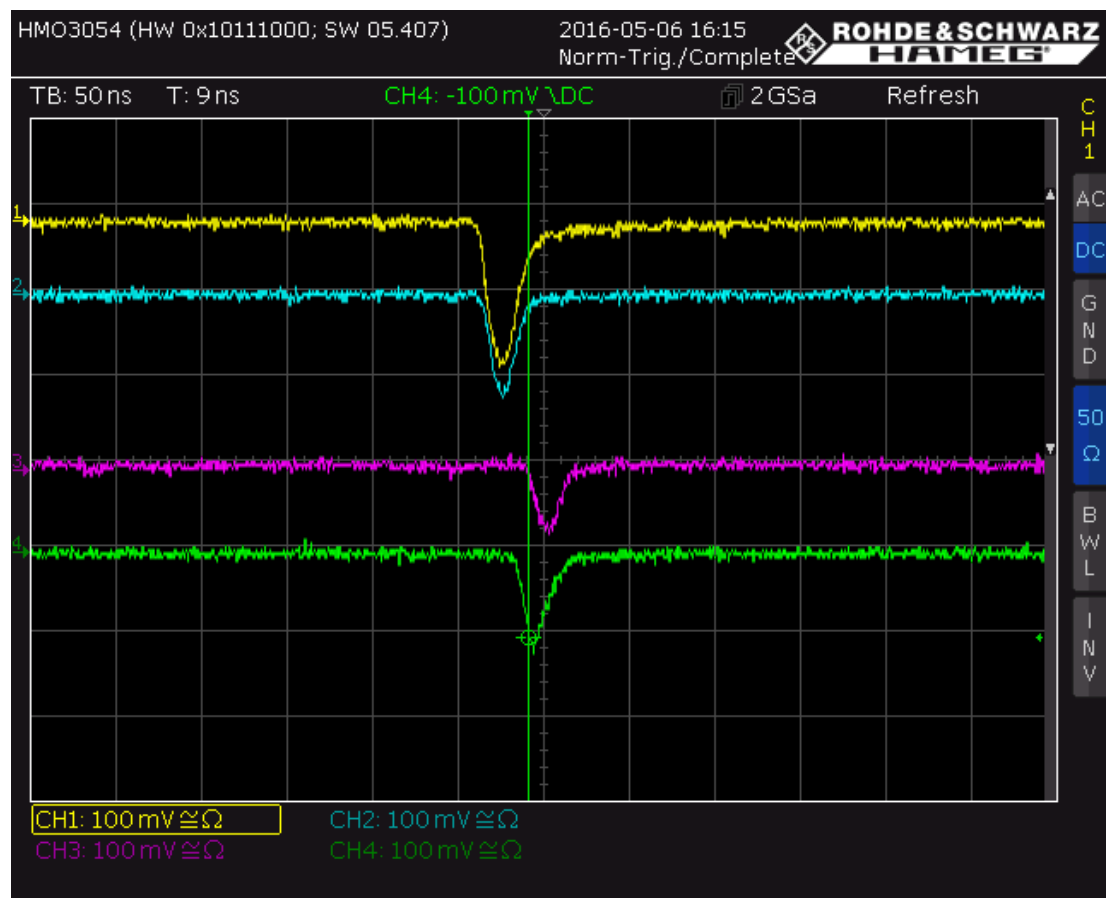

Figure 5. Typical signals after amplification from anode and cathode planes for operating conditions: Methylal pressure is 3.3 Torr, $+300 \mathrm{~V}$ on the anode, $0 \mathrm{~V}$ on the cathodes, and $-300 \mathrm{~V}$ on the guard planes. Trace 1 (yellow) and 2 (cyan) are signals from the anode planes of the top and bottom MWPC units. Trace 3 (magenta) and 4 (green) are signals from the two cathode planes of the top MWPC unit. The timescale is $20 \mathrm{~ns} /$ division and amplitude scale is $100 \mathrm{mV} /$ division. No polyethelene absorber has been placed between the Pu-239 and the Mylar collimator.

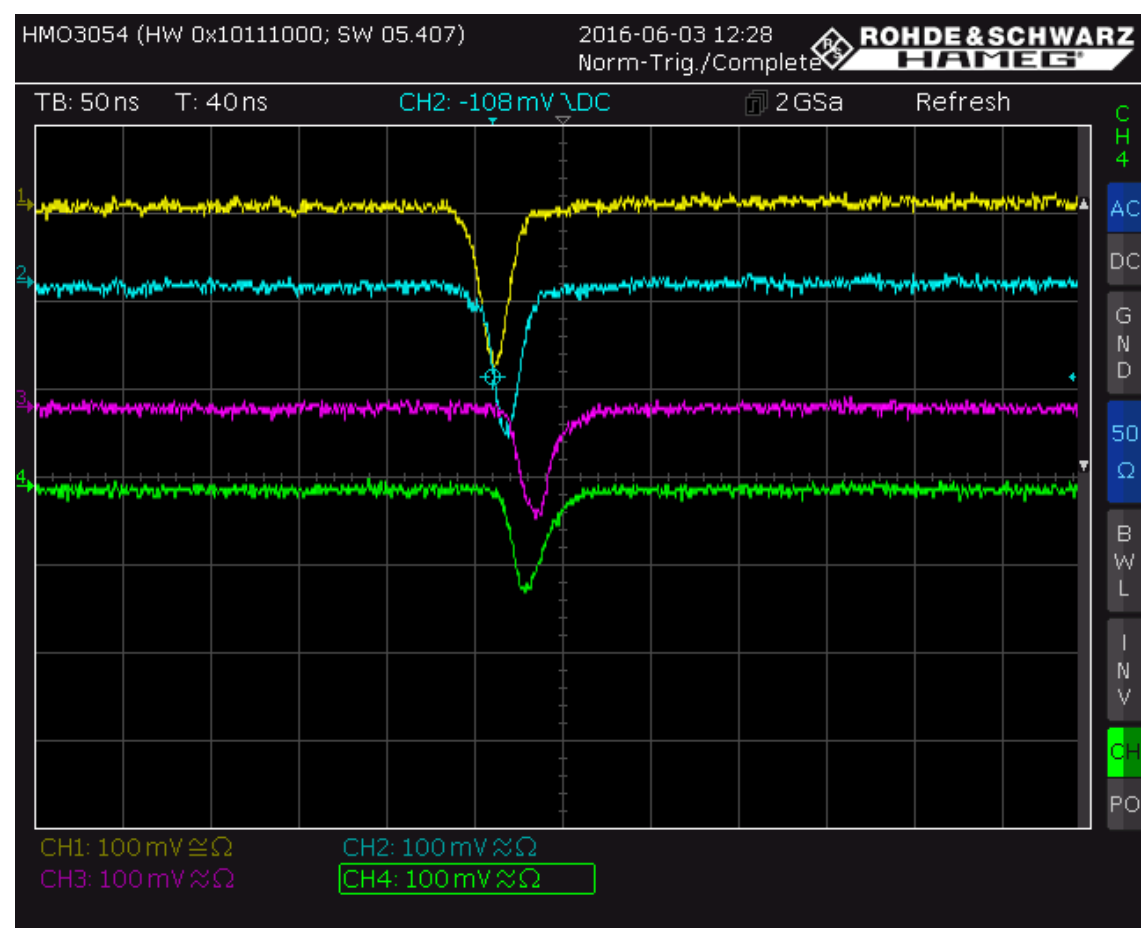

Figure 6. As in Figure 5 but with polyethylene absorber inserted between the Pu-239 and the Mylar collimator. 




Figure 7. The time difference distribution between signals from the anode planes of MWPC1 and MWPC2. The filling gas is methylal and the pressure is 3.0 Torr.



Figure 8. The same as in Figure 7 but with a $24 \mu \mathrm{m}$ polyethylene absorber inserted. Filling gas is hexane and pressure is 2.4 Torr.

When an avalanche occurs beside one or more anode wires of a MWPC, a positive charge is induced on several adjacent cathodes. The position of the avalanche can be determined by measuring the centre of charge of the induced charge distribution. In Figure 10, the time-difference spectrum between signals from the anode and that of the two cathode planes of MWPC1 is presented. The sigma of the Gaussian fit is about $1 \mathrm{~ns}$, which results in $1.5 \mathrm{~mm}$ position resolution ( $20 \mathrm{~ns}$ is equivalent to $30 \mathrm{~mm}$ ). A slightly better position resolution could be achieved using tap-by-tap signal amplitude readout and computation of the centroid via measured charge [9]. 


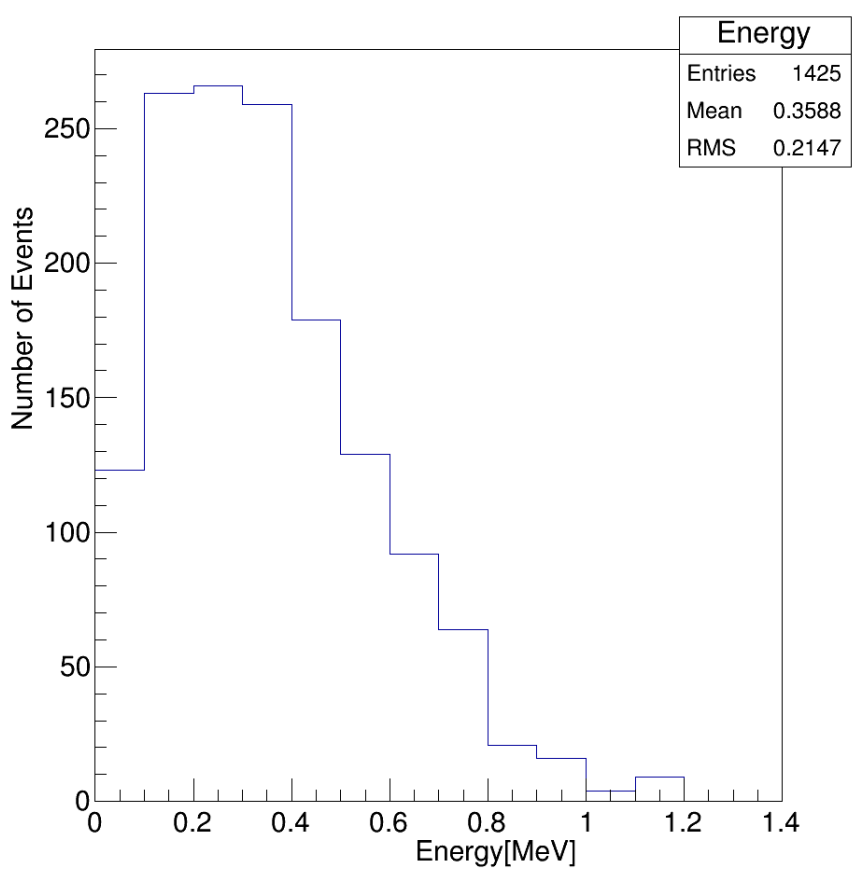

Figure 9. Energy distribution of $\alpha$-particles from $\mathrm{Pu}-239$ after passing of the $24 \mu \mathrm{m}$ polyethylene.

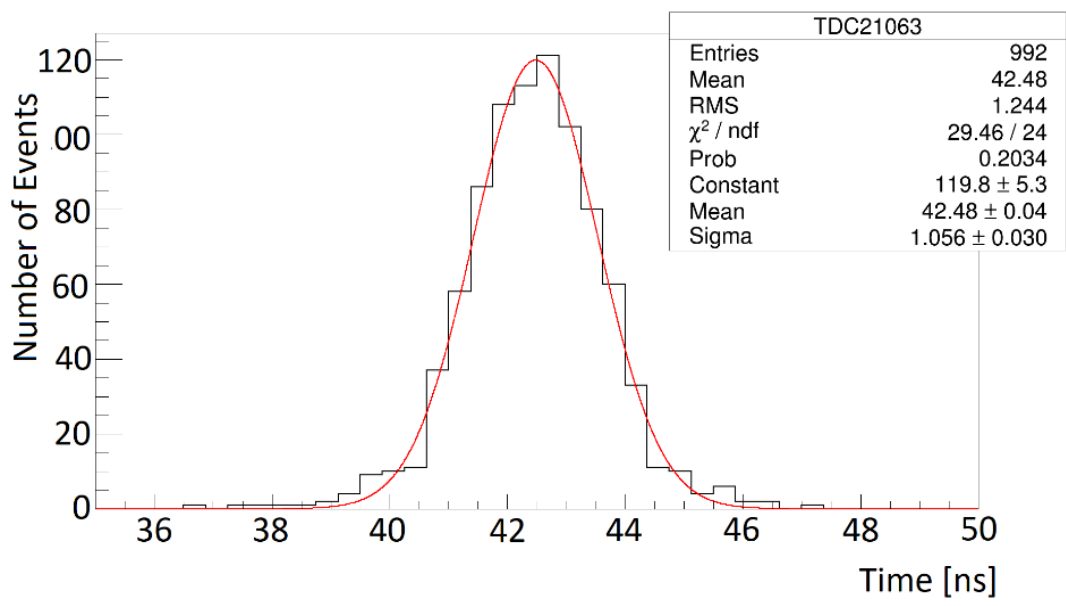

Figure 10. Time difference between the anode and one of two cathode planes of MWPC1. Filling gas is methylal and pressure is 3.0 Torr.

The time resolution of a MWPC unit-in terms of the width of the time-difference distribution between the anodes of MWPC1 and MWPC2-is displayed as a function of methylal pressure in Figure 11. It shows that the best time resolution can be achieved at a pressure lying in the range of 2-3 Torr. 


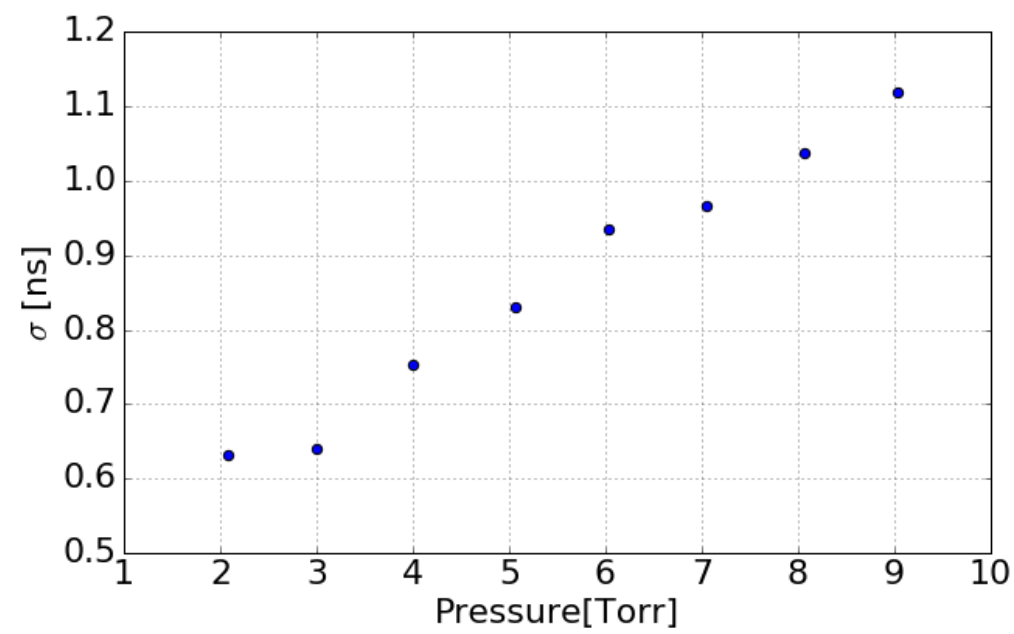

Figure 11. Width of the time-difference distribution for signals from the anode planes of MWPC1 and MWPC2 as a function of methylal pressure.

To be detected, low-energy decay particles require enough energy to pass through the module, i.e., they are required to have a range of at least $5.5 \mathrm{~cm}$ to generate signals in both MWPC units. In Figure 12, the electronic stopping powers (ionization energy losses) for $\mathrm{He}$ and $\mathrm{C}$ ions in methylal, simulated by means of the SRIM [12] software package, are displayed. The corresponding ranges of He and C ions in methylal, derived from the SRIM stopping powers, are presented in Figure 13. From Figures 12 and 13, it can be seen that an active-target detector system (Figure 3) filled to 3 torr methalyl is capable of detecting alpha particles with energies above $50 \mathrm{keV}$ and ${ }^{12} \mathrm{C}$ nuclei with energies higher than $100 \mathrm{keV}$. This is crucial for the detailed study of the photodisintegration of ${ }^{16} \mathrm{O}$ at energies $E_{\gamma}=8.0-8.5 \mathrm{MeV}$, where the $\alpha$-particles have energies of $\sim 0.6 \mathrm{MeV}$ and the ${ }^{12} \mathrm{C}$ nuclei have energies of $\sim 0.2 \mathrm{MeV}$. The respective $\alpha$-particle and ${ }^{12} \mathrm{C}$ ranges in methylal at 3 torr pressure are $0.3 \mathrm{mg} / \mathrm{cm}^{2}$ $(24 \mathrm{~cm})$ and $0.1 \mathrm{mg} / \mathrm{cm}^{2}(8 \mathrm{~cm})$.

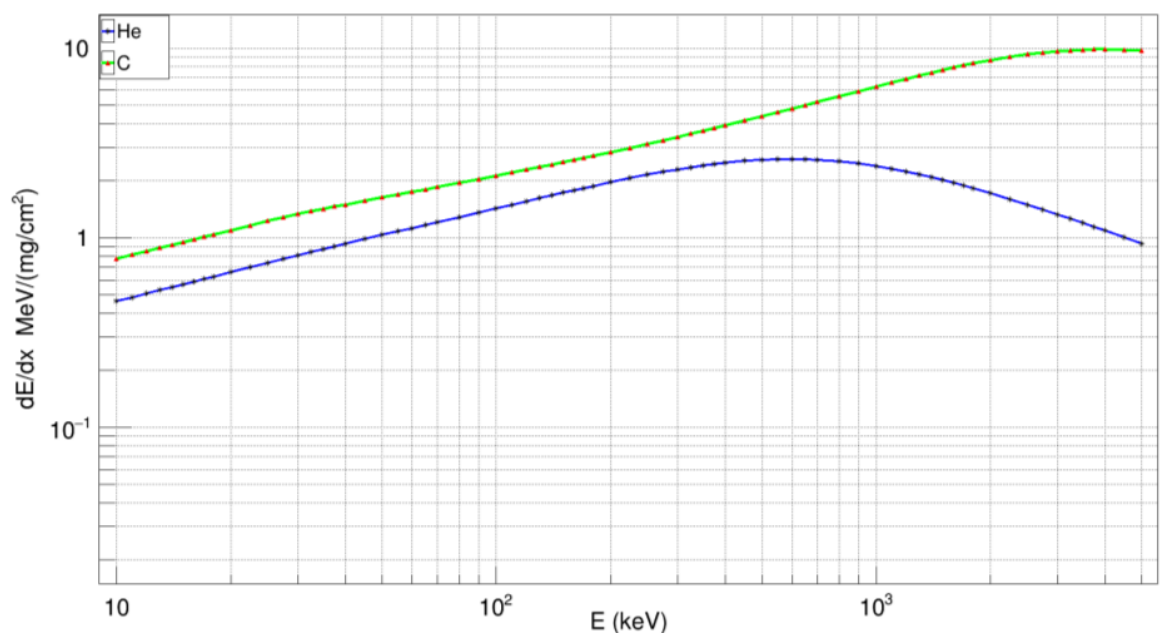

Figure 12. The electronic stopping power of $\mathrm{He}$ and $\mathrm{C}$ ions in methylal. 


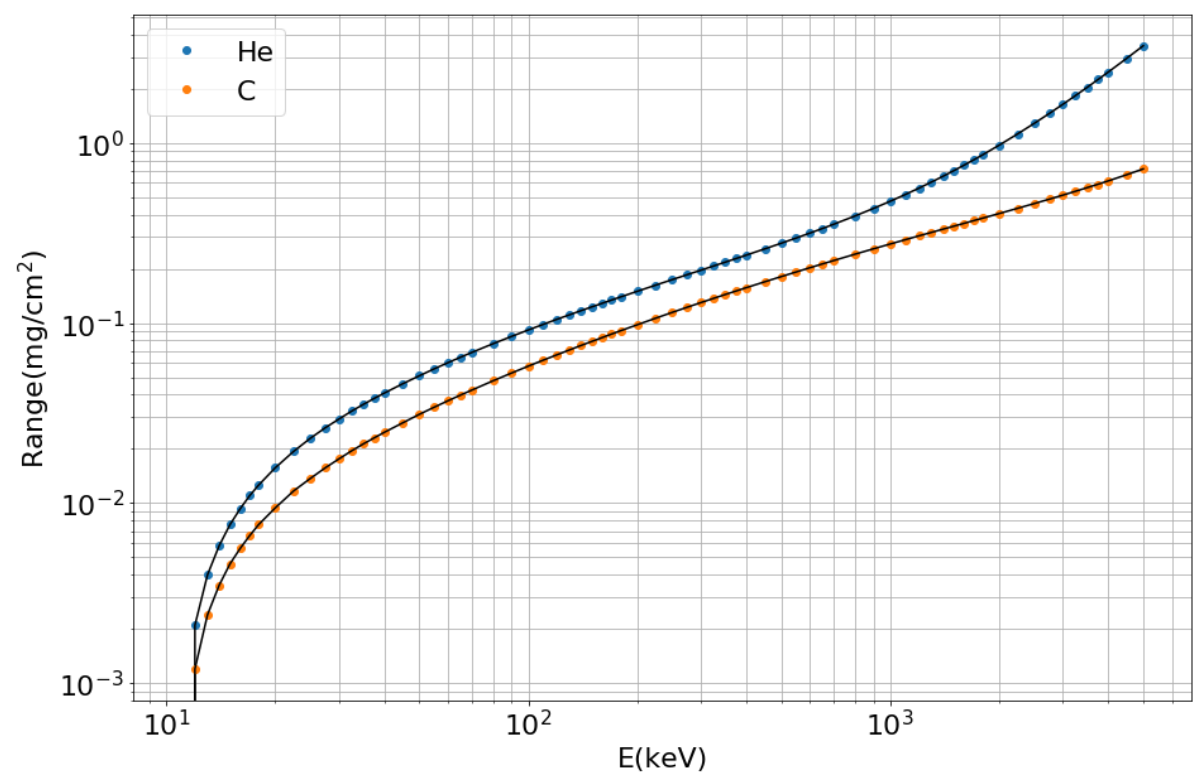

Figure 13. The ranges of $\mathrm{H}, \mathrm{He}, \mathrm{C}$ ions in methylal vs. energy.

The windowless MWPC units will be located as close as possible to the incident beams to maximize the geometrical acceptance and to minimize energy losses in the gas environment. It is expected that many background particles, e.g., due to electromagnetic processes, will be produced even though the sensitivity to electrons and photons is very low. Therefore, the rate capability of the MWPC units is a crucial factor. Typical signals from the anode plane of MWPC1 placed next to the ${ }^{239} \mathrm{Pu}$ source without any collimator are displayed over a time scale of around $12 \mu \mathrm{s}$ in Figure 14. From this, we infer that the MWPC has the capability to operate at rates of up to a few $\mathrm{MHz}$.

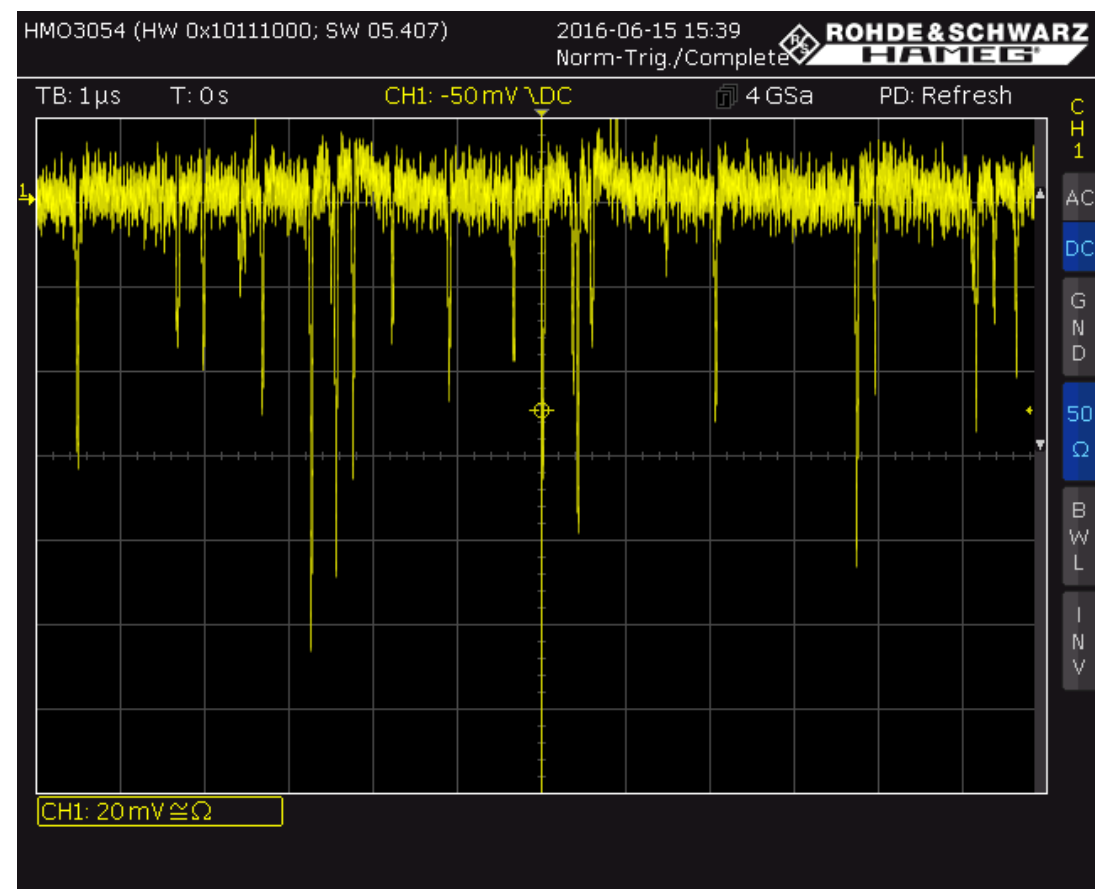

Figure 14. Typical signals from the anode plane of MWPC1 displayed on a $500 \mathrm{MHz}$ oscilloscope. The $\alpha$-particle source is ${ }^{239} \mathrm{Pu}$, with no collimation applied. MWPC conditions are hexane 2.85 Torr, $+300 \mathrm{~V}$ on the anode, $0 \mathrm{~V}$ on the cathodes, and $-300 \mathrm{~V}$ on the guard planes. 
Given that huge numbers of electron-positron pairs will be produced, the sensitivity of the low-pressure MWPC to minimum ionizing particles was measured by replacing the $\mathrm{Pu}$-239 $\alpha$-particle source with a $\beta$-particle source (Sr-90). The rejection factor for a single MWPC unit, i.e., the ratio of $\beta$-particle to $\alpha$-particle detection efficiencies is about $10^{-4}$. Since the ${ }^{16} \mathrm{O}$ photodisintegration event will be detected in four (1, 2, 3 and 4) MWPC units, the overall $\beta$-particle to $\alpha$-particle detection efficiency will reduce to $10^{-16}$. However, this rejection factor will be checked in dedicated experimental studies.

The energy resolution of $\alpha$-particles and ${ }^{12} \mathrm{C}$ nuclei depends on the L1, L2 and L3 dimensions (see Figure 1) and is expected to be in the range of $10-20 \%$. The time resolution for $\alpha$-particles with energies lying in the range $0.1-5.0 \mathrm{MeV}$ will be $\sim 0.45 \mathrm{~ns}$ and a flight time of $\sim 10 \mathrm{~ns}$. This will result in better than $10 \%$ energy resolution. In comparison, the bubble chamber experiment of Reference [3] provides no kinematic information, while the O-TPC experiment of Reference [4] provides only energy information but with a resolution several times better than the present proposed technique. However, in the present case, both angle and energy information will be recorded. Selection of back-to-back ${ }^{16} \mathrm{O}(\gamma, \alpha){ }^{12} \mathrm{C}$ reaction products will be used to reject background photodisintegration events from ${ }^{12} \mathrm{C}$ and ${ }^{16} \mathrm{O}$ nuclei, initiated by high-energy bremsstrahlung photons. This selection will also suppress any residual pair-production events. The separation of signal from background will be studied in realistic experimental conditions in future test experiments.

\section{Discussion}

A new oxygen active target is being developed for measuring ${ }^{16} \mathrm{O}(\gamma, \alpha){ }^{12} \mathrm{C}$ reaction cross-sections in the $8-10 \mathrm{MeV}$ photon energy region as produced by the LCB $\gamma$-ray beam of ELI-NP. It is based on the low-pressure MWPC technique and comprises two arms, each consisting of a pair of MWPC units. This allows the measurement of trajectories and velocities of ${ }^{16} \mathrm{O}(\gamma, \alpha){ }^{12} \mathrm{C}$ photodisintegration products, $\alpha$-particles and ${ }^{12} \mathrm{C}$ nuclei. The active area of the MWPC prototype units is $30 \times 30 \mathrm{~mm}^{2}$ but could be larger by a factor 2 .

At a pressure of a few Torr, methylal $\left(\left(\mathrm{OCH}_{3}\right)_{2} \mathrm{CH}_{2}\right)$ or hexane $\left(\mathrm{C}_{6} \mathrm{H}_{14}\right)$ serve as the working gases for MWPC operation, where hexane is intended for evaluation of the background from carbon nuclei in methylal. The time resolution of the MWPC for $\sim 5 \mathrm{MeV} \alpha$-particles is about 450 ps for methylal and $400 \mathrm{ps}$ for hexane. The corresponding position resolutions are $1.5 \mathrm{~mm}$ and $1.0 \mathrm{~mm}$, respectively. Both hexane and methylal, operated at 3 Torr, give an $\alpha$-particle detection threshold of about $50 \mathrm{keV}$, while for ${ }^{12} \mathrm{C}$ the threshold detection energy is around $100 \mathrm{keV}$.

We are planning to use this active-target technique at the LCB $\gamma$-ray facilities of HI $\gamma \mathrm{S}$, TUNL, Durham, USA [6] and ELI-NP, Magurele, Romania [7]. The advantages of this active-target system include good timing and position resolution, high rate capability, detection and identification of both photodisintegration fragments, and insensitivity to the electromagnetic background. The main disadvantage of this device is the low density of target nuclei, which is due to the low-pressure gas. This drawback can be compensated when using highly-directed, pencil-type LCB $\gamma$-ray beams by using a multimodule active-target system stacked up to a total length of $\sim 10 \mathrm{~m}$. In this way, the oxygen target thickness can be increased to $\sim 3 \times 10^{20}$ atoms $/ \mathrm{cm}^{2}$, giving a ${ }^{16} \mathrm{O}(\gamma, \alpha){ }^{12} \mathrm{C}$ reaction rate of $\sim 0.00135 \mathrm{event} / \mathrm{s}$. After event selection, the detected background rate from electron-positron pair production and other photodisintegration modes of ${ }^{16} \mathrm{O}$ and ${ }^{12} \mathrm{C}$ is expected to be negligible.

Author Contributions: A.M., J.R.M.A., D.L.B., B.V., K.L. and R.M. conceived physics motivation and the method; R.A. developed the detector; H.V. and P.K. developed the amplifiers; H.V and V.Ka. developed the DAQ; N.G. performed the experiments; V.Kh. and S.Z. analyzed the data; A.M., V.Ka. and J.R.M.A. wrote the paper.

Acknowledgments: This work is supported in part by Grant numbers 14CYC-1c11, 16-A1c66 and 15T-2B206 of the State Committee of Science, Republic of Armenia and by the UK Science and Technology Facilities Council, Grant numbers STFC 57071/1 and STFC 50727/1. D.L.B. is supported by the Extreme Light Infrastructure Nuclear Physics (ELI-NP) Phase II, a project co-financed by the Romanian Government and the European Union through the European Regional Development Fund - the Competitiveness Operational Programme (1/07.07.2016, COP, ID 1334). A.M. thanks to Ani Aprahamian for useful discussions.

Conflicts of Interest: The authors declare no conflict of interest. 


\section{References}

1. Fowler, W.A. Experimental and theoretical nuclear astrophysics: The quest for the origin of the elements. Rev. Mod. Phys. 1984, 56, 149-179. [CrossRef]

2. Weaver, T.A.; Woosley, S.E. Nucleosynthesis in massive stars and the $12 \mathrm{C}(\alpha, \gamma) 16 \mathrm{O}$ reaction rate. Phys. Rep. 1993, 227, 65-96. [CrossRef]

3. DiGiovine, B.; Henderson, D.; Holt, R.J.; Rehm, K.E.; Raut, R.; Robinson, A.; Sonnenschein, A.; Rusev, G.; Tonchev, A.P.; Ugalde, C. Bubble chambers for experiments in nuclear astrophysics. Nucl. Instrum. Methods Phys. Res. Sect. A 2015, 781, 96-104. [CrossRef]

4. Gai, M.; Ahmed, M.W.; Stave, S.C.; Zimmerman, W.R.; Breskin, A.; Bromberger, B.; Chechik, R.; Dangendorf, V.; Delbar, T.; France, R.H., III. An optical readout TPC (O-TPC) for studies in nuclear astrophysics with gamma-ray beams at HI $\gamma$ S1. J. Instrum. 2010, 5, P12004. [CrossRef]

5. Harutyunian, F.R.; Tumanian, V.A. The Compton effect on relativistic electrons and the possibility of obtaining high energy beams. Phys. Lett. 1963, 4, 176-178. [CrossRef]

6. Weller, H.R.; Ahmed, M.W.; Gao, H.; Tornow, W.; Wu, Y.K.; Gai, M.; Miskimene, R. Research opportunities at the upgraded HI $\gamma$ S facility. Progr. Part. Nucl. Phys. 2009, 62, 257-303. [CrossRef]

7. Filipescu, D.; Anzalone, A.; Balabanski, D.L.; Belyshev, S.S.; Camera, F.; la Cognata, M.; Constantin, P.; Csige, L.; Cuong, P.V.; Cwiok, M.; et al. Perspectives for photonuclear research at the Extreme Light Infrastructure-Nuclear Physics (ELI-NP) facility. Eur. Phys. J. A 2015, 51, 185. [CrossRef]

8. Vardanyan, H. Fast, low-noise amplifiers for low-pressure multi-wire proportional chambers. Proc. NAS RA SEUA Tech. Sci. 2017, LXX, 350-357.

9. Breskin, A. Progress in Low-Pressure Gaseous Detectors. Nucl. Instrum. Methods 1984, 196, 11-21. [CrossRef]

10. Assamagan, K.; Baker, K.; Bayatyan, G.; Carlini, R.; Danagoulian, S.; Eden, T.; Egiyan, K.; Ent, R.; Fenker, H.; Gan, L.; et al. Time-zero fission-fragment detector based on low-pressure multiwire proportional chambers. Nucl. Instrum. Methods Phys. Res. Sect. A 1999, 426, 405-419. [CrossRef]

11. Margaryan, A.; Adler, J.-O.; Brudvik, J.; Grigoryan, N.; Fissum, K.; Hansen, K.; Isaksson, L.; Knyazyan, S.; Lundin, M.; Marikyan, G.; et al. low-pressure MWPC system for the detection of alpha-particles and fission fragments. Armen. J. Phys. 2010, 3, 282-291.

12. James F. Ziegler. Available online: http:/ / www.srim.org (accessed on 30 January 2018). 